\title{
El coeficiente ureosecretorio de Ambard
}

\author{
POR el DR. MANUEL A. VELASQUEZ \\ Profesol de Quimica en la Pacultad de Medicina
}

Estudiando la función renal desde el punto de vista secretorio, se encuentra que dos actos fundamentales la caracterizan: la eliminación del agua y la eliminación de las sustancias disueltas. Ambos, sufren modificaciones patológicas que aunque conexas parecen relativamente independientes y dan lugar en la clínica a dos sídromas: el sindroma hidropígeno y el sindroma uremígeno.

Dejando de lado el estudio de la eliminación acuosa que se aprecia por la prueba de la poliuria experimental de Albarran, nos concretaremos a estudiar la eliminación solida formada principalmente por la urea.

Durante mucho tiempo se creyó que el simple dosage de 12 urea en la orina bastaba para poseer datos seguros sobre el funcienamiento del riñon, pero después se vió que esta apreciación tenía un valor semiológico muy limitado, ya que la cifra encontrada por el dosage está a merced del grado nitrogenado de la alimentación o del trabajo ureopoiético de la célula hepática, no traduciende. por consiguiente, de manera indudable, perturbación de la secreción renal.

Estudiando la nefritis, WIDAL (1) puso de manifiesto que el dosage de la urea contenida en la sangre proporcionaba datos de mayor valor sobre el funcionamiento renal, que el simple dosage de la urea urinaria, por cuanto, dentro de ciertos límites, la cantidad de urea contenida en la sangre, traduce la acumulación de esta sustancia a consecuencia de la disminución del poder de depuración del riñón.

(1) Wival.-Le pronostic dars le mal de Bright par le dosage de trerée du sant -Societe Medicale des Hopitaux.-Paris, pág. 627 - 1911. 
Estos estudios que hicieron decir a WIDAL (1) que el dosage de la urea en la sangre era un elemento indispensable para todo médico que trate una nefritis, y que gracias a él, se poseía un elemento de certeza para predecir su evolución y su pronóstico, fueron bien pronto amenguados en su valor absoluto, porque estudiando las variaciones de la urea en la sangre se comprobó que se encontraban bajo la dependencia del grado de funcionamiento renal, de la riqueza nitrogenada de la alimentación, del estado de los órganos uropoiéticos y de la nutrición en general.

Las variaciones dependiendo del régimen alimenticio, fueron reconocidas por el mismo WIDAL y por JAVAL, quienes sustituyeron el concepto de cifra absoiuta de urea en la sangre por la noción de índice de retención ureica, que traduce la relación entre el nitrógeno alimenticio y la azohemia.

La deficiencia renal no puede tampoco medirse por el grado de uremia, pues, si los datos son exactos cuando se trata de azohemias leves, en los casos de azohemias extremas, que debían traducir siempre lesiones irreparables, no siempre se trata de esto. Así, las lesiones unilaterales poco afectan la azohemia, apesar de que sean graves, pues, hay función supletoria del otro riñón, cosa que CHEVASSU (2) ha comprobado en la mayoría de los urinarios quirúrgicos; de otro lado hay hiperazohemias que no traducen una lesión irreparable del riñón, como la observación señalada por MosNy y Javal (3) de un caso de nefritis saturnina que sobreviví quince meses con una azohemia de 4,22 grms.; como las azohemias encontradas por GUY LAROCHE y BRODin (4) en el curso de nefritis agudas benignas, sobrevenidas durante la evolución de la neumonia; en fin, NoBecourt y Bidot (5) refieren un caso de nefritis impetiginosa infantil en el cual la azohemia alcanzó a 6,17 grms. sin que fuera mortal, pues, descendió después a despecho de la persistencia de la albuminuria y hematuria.

La influenria de los órganos ureopoiéticos también disminuye el valor semiológico de la azohemia, por cuanto la urea puede provenir de la desasimilación de los tejidos, como sucede en los estados de inanición, febriles, etc, sin que haya ingestión introgenada, y la elaboración ureica depende, en último término, del funci namiento hepático.

(1) WIDAL. - Les grands s?mdromes fonctionnels du mal de Bright.- ILa Presse Medicales. 20 novembre 1912 .

(2) Mauricie Chevassu.- Le dosage de l'urée sanguine et la constante uremique.

chex les urinaires chirurgicales._LL Presse Medicales, 1912 pág. 514.

(3) Mosny et Javal-Crise de grande azotemie.-Parls Medical-1913 pág.358.

(4) GUY IAROCHE ot BRODIN.-Azotemie aigue au cours de quelques infections,

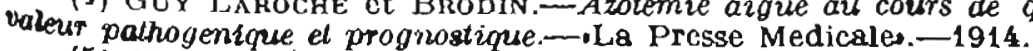

(5) NoBEcount el BIDOT. - Grand azotemte passagete au cours dine nephrte atgue. - ila Presse Medicale.-1912. 
Restringido así el valor de la azohemia como indicador del grado de insuficiencia renal, se pens6 que podía suministrar datos de positivo valor la comparación de la urea de la sangre, con la urea de la orina. Aunque esta investigación fué hecha por primera vez por GRENANT en 1904, corresponde a AMBARD haber señalade y demostrado su importancia, así como la constancia de sus resul. ádos y el valor de la relación de la urea sanguinea con la urea de la orina, por lo cual se conoce a esta relación con el nombre de constante o coeficiente de AMBARD.

Este es el únicométodo perfecto para conocer la función ureica del riñon puesto que permite comparar, durante un mismo periodo de tiempo, la urea que entra en el riñon, esto es, la urea sanguíne y la urea que sale, esto es, la urea de la orina. Como el riñón es d único órgano interpuesto, las variaciones de la reiacion

$$
\frac{\text { urea de la sangre. }}{\text { urea de la orina. }}
$$

indicarán forzosamente las modalidades del funcionamiento ureico del riñón.

Fundamentos del método de AMBARD.-La secreción renal de la urea obedece siempre, en el hombre sano como en el enfermo de Jesiones uremígenas, a dos hechos constantes, que tienen el caracter de otras tantas leyes:

I. La cantidad de urea eliminada crece en razon directa de la que contiene la sangre, si ésta aumenta, la otra se exagera.

I1. Si se exagerala diuresis, como ocurre en la poliuria expontánea o en la experimental, la concentración ureica de la orina disminuye, pero la cantidad total de urea eliminada aumenta en definitiva.

Partiendo de estos hechos perfectamente comprobados, АмBARD (1) trató de conocer el mecanismo exacto de la secrecion y deducir la medida de sus variaciones cuando las lesiones del parénquima renal rompen el equilibrio normal y este estudio lo conduj• a sostener en 1910, las siguientes leyes de la secreción renal ureica.

la Ley. - "Cuando el riñón segrega la urea a concentración $u$ : $i$ naria constante (C), la cantidad de urea el minada (D), varia en razb́n directa del cuadrado de su concentración en la sangre (Ur) H. - Esto es' lo que expresan las siguientes formulas de AMBARD:

$$
\frac{D}{D,}=\frac{U r^{2}}{U r^{2}} \circ \text { lo que es igual } \frac{V \bar{D}}{\sqrt{D^{\prime}}}=\frac{U r}{U r^{\prime}}
$$

(1) AmBARD. - Rapports cntre le teaux de l'uree dans le sang el l'eltmination de l'uree dans Curine.-Comptes Rendus de la Societé de Biologie - París, 10 Nov. 1010. 
de donde

$$
\frac{\mathrm{U}_{\mathrm{r}}}{\sqrt{\mathrm{D}}}=\frac{\mathrm{U}_{\mathrm{r}^{\prime}}}{\sqrt{\mathrm{D}^{\prime}}}=\ldots \ldots \ldots \mathrm{K} \text {, cifra constante. }
$$

Esto quiere dedcir que la cantidad de urea eliminada por el rinoon (D), varía siempre en razón directa del cuadrado de concentración de la urea de la sangre (Ur).

2a. Ley.- "Cuando la concentración de la urea en la sangre (Ur) es constante, la cantidad de urea eliminada (D), varia en razón inversa a la raiz cuadrada de su concentración en la orina (C).-Esto se expresa por las fórmulas respectivas:

$$
\begin{gathered}
\frac{D}{D^{\prime}}=\frac{\sqrt{C^{\prime}}}{\sqrt{C}} \text { osea } D \times \sqrt{C}=D^{\prime} \times \sqrt{C^{\prime}} ; \text { de donde } \\
D^{\prime}=D \times \frac{\sqrt{C}}{\sqrt{C^{\prime}}}
\end{gathered}
$$

Esta fórmula quiere decir que la cantidad de urea eliminada (D) varía inversamente a la raíz cuadrada de la concentración (C).

3a. Ley-a Si las concentraciones ureicas de la sangre y de la or $i$ na son variables, la cantidad de urea eliminada por el riñón (D), varía en ploporción directa al cuadiado de su concentración en la sangre (Ur) y en proporción inversa a la raiz cuadrada de su concentracion en la orina (C). - - Las siguientes formulas expresan esta ley:

de donde

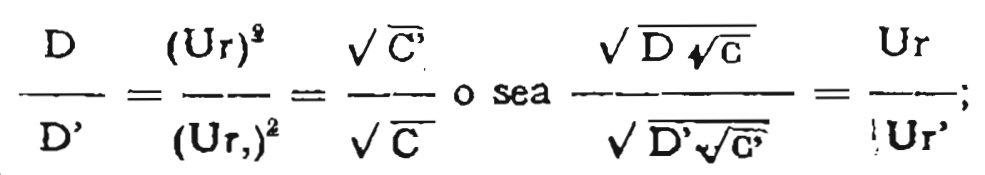

$$
\frac{\mathrm{Ur}}{\sqrt{\mathrm{D} \vee \overline{\mathrm{C}}}}=\frac{\mathrm{U} r^{\prime}}{\sqrt{\mathrm{D}^{\prime} \sqrt{\mathrm{C}^{\prime}}}}
$$

Según esta formula se pueden comparar la concentración ureica de la orina (C) y la de la sangre (Ur) y observar que la urea segregada por el riñón (D) varía a la vez, en razón directa al cuadrado de su concentración en la sangre y en razón inversa a la raíz cuadrada de su concentración en la orina.

En resumen, se puede decir que: La secreción renal de la urea, en un individuo cualquiera y en cada momento de su vida, es proportional al cuadrado de la concentración ureica de la sangre e inversa- 
mente proporcional a la rafz cuadrada de la concentración ureica de la orina; lo que se expresa por la siguiente formula algebraica.

$$
K=\frac{U_{r}}{\sqrt{D \sqrt{C}}}
$$

en la cual la letra $\mathrm{K}$ designa una cifra que es siempre constante, que mide la función ureica del riñón.

Esta formula es muy general, y para poder referir los datos de cada caso particular, AMBARD ha tenido que tomar en cuenta el pes. del individuo y calcular la cifra que se obtenga de $D$, refiriéndola a la que correspondería, a un peso tipo convencional $P$ que se ha fijado en 70 kilogramos, de donde resulta $D \times \frac{70}{\mathrm{P}}$ que es una cifra mas aproximada a la realidad.

lgualmente ha tenido que convenirse en fijar para la concentración urinaria $C$ un tipo uniforme, que se ha resuelto sea 25 por 1000 , tanto porque la concentración urinaria normal oscila al rede. dor de esa cifra, como porque es muy cómoda para los cálculos la cifra 5 de su raíz cuadrada.

Según ambas correcciones, la fórmula definitiva de la constante ureica de $A$ MARD, es la siguiente:

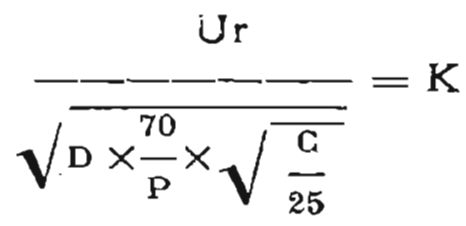

Técnica para el exámen de la "Constante ureican de AMBARD.Se procura recoger la orina en el espacio de media hora y tomar simultaneamente en este mismo periodo de tiempo, una cierta cantidad de sangre para dosar en ella la urea.

Para lo primero (recolección de la orina) se comienza por evacuar completamente la vejiga, ya por la micción o lo que es más seguro por cateterismo; en el momento en que la vejiga está completamente vacía, se anota la hora exacta; diez minutos después se aplica al paciente dos ventosas escarificadas con el objeto de recojer unos cuarenta gramos de sangre. Al cabo de media hora se recoje la orina que se ha acumulado en la vejiga, a notando con toda exactitud la hora en que corren las últimas gotas de orina.

Se efectúa después la dosificación de la urea sanguínea, previa separación del suero y precipitación subsecuente de las albúminas. 
En la orina también se dosifica la urea, despues de apreciar e! volúmen de la orina recojida, determinandose en seguida la urea eliminada en 24 horas.

Sustituyendo en la fćrmula definitiva los dacos obtenidos en el sujeto que se examina, se conocerá su constante ureo secretoria.

Valor numérico de $K$ en estado normal. - El coeficiente azohemico normal, está en gran parte a merced de los procesos a nalíticos utilizados, sobretodo para el dosaje de la urea en la sangre y también en razón del metabolismo individual. AMBARD admite que la constante en el individuo normal oscila entre $0,065,0.080$ y 0,090 , pero la cifra que ha adoptado es $K=0,065$. Alberto de. Aguiar (1) profesor de Urolngía en la Facultad de Medicina de Porto (Portugal) da como valor medio provisorio $K=0,090$ pues, sus análisis le han revelado oscilaciones entre 0.070 y 0,110 .

Consideraciones generales acerca de la "constante" patológıca.Aceptada la cifra media de $\mathrm{K}=0,065$, se observa que, varía en los diferentes estados patológicos en los cuales la función renal secretoria se altera; así las cifras de $\mathrm{K}$ son superiores a la normal y se acercan a la unidad a medida que crece la deficiencia renal provocada por las lesiones uremígenas. Gracias a la constante ureo-secretoria de AMBARD, se aprecia numericamente el quantum de la insuficiencia renal respecto a la urea.

Se admite, que a partir de 0,080 la secreción renal comienza a ser imperfecta; que las cifras comprendidas entre 0,090 y 0,125 acusan ya insuficiencia ureica evidente y que ésta es muy grave con cifras de 0,200, hasta llegar a la impotencia funcional absoluta cuando se encuentran cifras que se acercan a 0,500 .

En las lesiones hidropígenas puras, en cambio, la constante ofrece cifras inferiores a las normales.

Por último, la constante ureica de AMBARD ha demostrado que no pueden tomarse como dato absoluto las cifras dela uremia, pues, en sujetos cuya cifra de uremia, eran $0,50,0,40$ y aún 0,30 grms. de urea por litro de sangre, la "constantes se ha revelado patol6gica. Las investigaciones hechas a este respecto han revel ado que el paralelismo entre las cifras de la wuremias y las de $?_{2}$ - constante desaparece cuando la urea de la sangre se encuentra por arriba de 0,50 gr. por litro.

Los datos que la constante ureica de AmBARD suministra en Cirugía son interesantes; sin embargo, en muchas afecciones quirúrgicas de los riñones, por ejemplo, se encuentra una "constante" normal, lo cual indica que el otro riñ́n compensa la función del

(1) Alberto Aguiar.- O coeficiente azotemico ou ureio-secretorio dos Drs. Ambard e Moteno.-aRevista de Semiotica laboratorialo. - Porto 1916. 
atacad., como acon ece en la tuberculosis renal unilateral; mien. tras que la "constante" aparece aumentada cuando los dos riñones están atacados.

Semiologicamente, el coeficiente ureosecretorio, puede tam. bien dar idea aproximada del valor de la actividad renal existente y por consiguiente de la actividad renal perdida, según que los valores de $\mathrm{K}$ ' sean mayores que los normales.

Hay muchos factores que falsean el valor de los datos suminis. trados por la constante de AMBARD, citaremos la recolección imperfecta de la orina, las orinas de glicosúricos, edematosos, asciticos y asistólicos, en una palabra, las orinas de los grandes oligúricos. poliúricos elevados o febriles.

Además, se tendrá presente las perturbaciones renales pasajeras de orden reflejo, antes de concluir definitivamente sobre el valor de las cifras obtenidas.

Modificaciones a la constante de AMBARD. - Lo corriente y practico es referir todo resultado de análisis a 100; con éste propó. sito Rodillon (1) propone simplificar la fórmula de AmBARD, de manera que con las cifras normales se obtenga un coeficiente igual a 100

1)-En la fórmula $K=\frac{U r}{\sqrt{\mathrm{D} \times \frac{70}{\mathrm{P}} \times \sqrt{\frac{\mathrm{C}}{25}}}}$ el denominador es muy superior al numerador, de manera que para aproximar el cuociente a la unidad se invertirá la fracción, quedando la formula así:

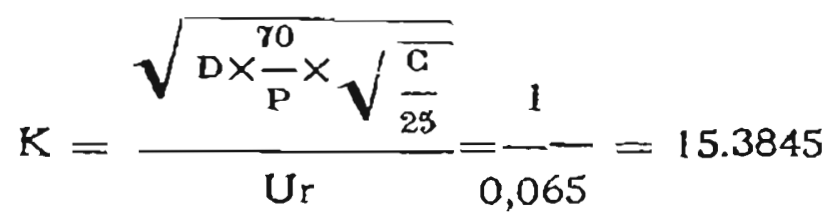

Para transformar esta relación igual a 100 será necesario multiplicar el numerador por un número $\mathrm{X}$ por 15.3845 .

o mas simplemente 6.5

$$
X=\frac{100}{15.3845}=6.5001
$$

(1) Rodillon.- Journal de Pharmacie et de Chimiques.-Paris 1912. 
Designando la constante $\mathrm{K}$ por $\mathrm{N}$, la fórmula será:

$$
N=\frac{0.5 \times \sqrt{\mathrm{D} \times \frac{70}{\mathrm{P}} \times \sqrt{\frac{\mathrm{C}}{25}}}}{U r}
$$

2) - Para simplificar está formula se hace pasar el factor 6.5 bajo el radical, elevándole al cuadrado, lo que dá:

$$
\mathrm{N}=\frac{\sqrt{6.5^{2} \times{ }_{\overline{\mathrm{P}}}^{7} \times \mathrm{D} \times V_{25}^{\bar{C}}}}{U_{\mathrm{r}}}
$$

Esto es igual a $\mathrm{N}=\frac{\sqrt{\mathrm{p}} \times \frac{\mathrm{4}}{\mathrm{4}} \times \sqrt{\frac{\mathrm{C}}{25}}}{\mathrm{U}_{\mathrm{r}}}$

$$
\mathrm{Ur}
$$

Efectuando la operación de $42,25 \times 70=2,957$ la fórmula será:

$$
N=\frac{\sqrt{\frac{2.957}{P} \times D \times \sqrt{\frac{G}{25}}}}{U r}
$$

Como sería muy dificil retener un factor tan grande y tan complicado el autor ha redondeado la cifra reemplazandola por $\mathbf{3 0 0 0}$ lo que da:

$$
N=\frac{\sqrt{\frac{3000}{\mathrm{P}} \times \mathrm{D} \times \sqrt{\frac{\mathrm{C}}{25}}}}{U_{r}}
$$

3)-Otro término de la formula que se simplifica es el peso $P$ que se expresa en kilógramos; puede sin cambiar su valor expresarse en unidades diferentes; así puede expresar $41 \mathrm{k} 50060.0415$, corriendo la coma 3 cifras hacía la izquierda, es decir, se habrá dividido por 1000 , el peso expresado en kilogramos.

Ahora, dividiendo po 1000 el denominador $P$, para no cambiar nada en la fracción, debemos dividir el numerador por el mismo número, operaciones que no alteran el valor de ella. 
La fórmula se habrá simplificado aún mas quedando así:

$$
N=\frac{\sqrt{\mathrm{D} \times \frac{3}{\mathrm{P}} \times \sqrt{\frac{\mathrm{C}}{25}}}}{\mathrm{Ur}_{\mathrm{r}}}
$$

Quedará pues la fórmula simplificada con los siguientes valo. res:

$\mathrm{N}$, constante $=100$

$\mathrm{C}$, concentración ureica de la orina por mil

Ur, concentración ureica de la sangre por mil.

$\mathrm{D}=$ rendimiento ureico de la orina en 24 horas

$P=$ peso del sujeto expresado en gramos $\left(\frac{1}{1000}\right)$ del peso en $\mathbf{k m s}$

La formula definitiva se expresará así:

$$
N=\frac{\sqrt{\frac{3 D}{P} \times \sqrt{\frac{\mathrm{C}}{25}}}}{U_{r}}
$$

La fórmula propuesta por Rodillon es:

10.- Simple y fácil de retener.

20.--Respeta integramente todos los datos de AMBARD.

$3^{\circ}$. - Da una indicacicin precisa a primera vista. Así suponiendo un caso, tendríamos la fórmula:

$$
N=\frac{\sqrt{\frac{3 \times 5.96}{0.015} \times \sqrt{\frac{2.16}{25}}}}{0.187}=60 \%
$$

en la cual se dirá que la permeabilidad renal es igual a $60 \%$ de 10 que es al estado normal.

4)-La cifra de la normal es 100 y corresponde exactamente a 0.065 que es la normal de AMBARD.

Para los que están habituados al coeficiente de AMBARD 0.065 sería fácil obtener éste valor de la constante $K$ de AMBARD, aplicando la fórmula muy simple: $\mathrm{K}=\frac{6.5}{\mathrm{~N}}$

Ejemplo: se desea saber cual sería el valor de 60 correspondiente a $N$ (coeficiente RODILLON) si hubiera sido calculado el coeficiente conforme a la fórmula de AMBARD; bastará aplicar 
la formula anterior, reemplazando $\mathrm{N}$ por el valor obtenido. $K=\frac{6.5}{60}=0.108$ será la constante dada por la fórmula de AMBARD.

MC. Lean (1) después de estudiar la relación entre el nitrogeno ureico y el no ureico contenido en la sangre y en la orina y de ponderar el valor que tiene la cifra arbitraria de la constante ideal, propone el siguiente índice de excreción, que está formado por la excreción de la urea en 24 horas (D), multiplicada por la raíz cuadrada de la concentración de ia urea de la orina (C), multiplicado por una constante $(8,96)$, dividido todo por el peso del cuerpo del. sujeto (Wt), multiplicado por la concentración de la urea en la sangre elevada al cuadrado $\left(\mathrm{Ur}^{2}\right)$

La fórmula del índice es la siguiente:

$$
I=\frac{D \times V}{W t \times U r^{2}}
$$

El índice (2) se deduce basándose en los postulados de AMBARD.

En efecto, según las leyes de AMBARD:

$1 \quad K=\frac{U r}{\sqrt{D}} \quad\left(\frac{U r}{0.08}\right)^{2}$

$20.08=\frac{\mathrm{Ur}}{\sqrt{\text { Exc.normal }}} \quad$ Exc. normal $=\left(\frac{\mathrm{Ur}}{\overline{0.08}}\right)^{2}$

$3 \frac{\text { Excreción }}{\text { Exc. normal }}=\frac{\left(\frac{U r}{K}\right)^{2}}{\left(\frac{U r}{0.08}\right)^{2}}=\left(\frac{0.08}{k}\right)^{2}$ (1) Franklin C. Mc. LFAN. - Clinical delermination of Renal Function by an
Index of Urea Excretion. - The Journal of the American Medical Association. Vol LXVI. No. 6- 1916.

(2) El cálculo del indice se simplifica mucho mas, empleando la regla graduada ad hoe, que han fabricado Keuffel y Esser Co, 127 Fuiton Street, New York. 
4 Indice $=100 \times\left(\frac{0.08}{K}\right)^{2}=\left(\frac{0.08}{K}\right)^{2}$

Sustituyendo por K (coeficiente de AMBARD) y simplificand. se tiene:

$$
\begin{aligned}
& \text { Crm. Urea p. } 24 \text { horas } \vee \text { Gms. Urea por litro orina } \times 96
\end{aligned}
$$

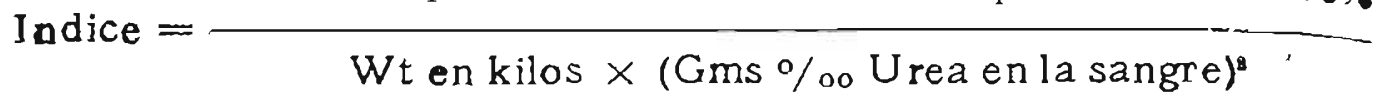

$$
\begin{aligned}
& \text { Cuando } \mathrm{K}=0,08 \text { tipo normal. } \\
& I=100
\end{aligned}
$$

Apreciaciones y resultados sobre el indice ureosecretorio. - El índice es independiente: de la ingestión del nitrógeno.

El índice normal ideal es 100 .

Comparando con el coeficiente de ANBARD: valores inferiores a 80 , que corresponden a un coeficientre de AMBARD de 0.090 , no se obtienen nunca en individuos normales, los sujetos no normales tienen unindice siempre inferior a 100. Los valores superiores a 100 sor la regla, pudiendo llegar a 180 y 200.

Se pueden observar valores más altos en ciertas condiciones patológicas asociadas a un aumento de excreción ureica.

Un índice bajo, puede ser solo temporal, como en la congestion pasiva, consecuencia de la disminución de la energía del corazón, así como también en cierlas nefritis; en tales casos se vuelve al índice normal, mejorando las condiciones que produjeron esa insuficiencia renal.

De un modo general índice bajo, persistente, es indicacifn de una enfermedad renal seria.

Un índice bajo asociado a disminución de la excreción de ori. na, con alta concentración de urea, sugiere un estado congestiv pasivo del riñón.

El mismoíndice con excreción de orina normal o aumentada, con concentración de urea disminuida, es característico de las nefritis.

Un índice inferior a 80 es anormal, aunque no es necesariamente grave.

En las enfermedades renales uri indice inferior a 50 es indicador de una alteración profunda. 
La lesión del riñón está en razón inversa del índice: mayor es la alteración, menor es el indice y se acerca a cero.

El índice no tiene valor cuando la eliminacion urinaria es inferior a 500 c. c. en 24 horas.

En la determinación del índice no debe olvidarse de disminuir 12 cifra correspondiente al amoníaco preformado; de aquí que sean indispensables tres dosages: urea en la sangre y en la orina y amoníaco en la orina. 